\title{
Generación de mesoporosidad en zeolitas ZSM-11, BETA e Y por tratamiento alcalino
}

\author{
Eliana Diguilio ${ }^{1}$, Agostina Córdoba $^{1}$, Candelaria Leal Marchena ${ }^{1}$, \\ María Soledad Renzini ${ }^{1}$, Liliana Pierella ${ }^{1}$
}

\footnotetext{
${ }^{1}$ Centro de Investigación y Tecnología Química (CITeQ), UE CONICET- Universidad Tecnológica Nacional, Facultad Regional Córdoba, Maestro López esq. Cruz Roja Argentina, Ciudad Universitaria, (5016) Córdoba, Argentina e-mail: ediguilio@frc.utn.edu.ar
}

\section{RESUMEN}

Las zeolitas Y, ZSM-11 y BETA, ampliamente usadas en refinerías y procesos petroquímicos, química fina y procesos ambientales fueron sometidas a un tratamiento alcalino de desilicación con el fin de generar mesoporosidad en la estructura. El proceso utilizado consistió en tratar las muestras con una solución acuosa de $\mathrm{NaOH}$ a $65^{\circ} \mathrm{C}$ durante 30 minutos. Luego se realizó un intercambio con $\mathrm{NH}_{4} \mathrm{Cl}$, para restablecer la acidez, seguido por desorción en corriente de $\mathrm{N}_{2}$ y posterior calcinación a $500^{\circ} \mathrm{C}$.

Las muestras tratadas fueron evaluadas por diferentes técnicas tales como DRX, ICP, SEM, área superficial por método BET, área específica externa, volumen de mesoporo y distribución de tamaño de poro por el método BJH.

Los tratamientos realizados produjeron en los materiales zeolíticos una fase mesoporosa con arreglo hexagonal y tamaño de poro definido, coexistiendo con la fase cristalina remanente. La generación de mesoporos, causaría una disminución de la relación $\mathrm{Si} / \mathrm{Al}$, acompañada de un aumento del área superficial con respecto al material de partida. Las especies Si son extraídas selectivamente, aunque también se remueven especies de Al. Se observó también un aumento del diámetro de poro y volumen de mesoporo, conservándose la estructura microporosa de las matrices de partida.

Palabras clave: Zeolitas, Mesoporosidad, Caracterización, Glicerol.

\section{ABSTRACT}

Zeolites, Y, ZSM-11 and BETA, widely used in refineries and petrochemical processes, fine chemistry and environmental processes were subjected to an alkaline treatment of desilication, in order to generate mesoporosity in the structure. The process used consisted of treating samples with an aqueous solution of $\mathrm{NaOH}$ at $65^{\circ} \mathrm{C}$ for 30 minutes. An exchange with $\mathrm{NH}_{4} \mathrm{Cl}$, to recover the acidity, followed by desorption in a $\mathrm{N}_{2}$ stream and subsequent calcination at $500^{\circ} \mathrm{C}$ was then performed.

The treated samples were evaluated by various techniques such as XRD, ICP, SEM, and surface area determination by BET method, specific external area, mesopore volume and distribution of pore size by the BJH method.

This treatment resulted in a mesoporous zeolitic materials phase hexagonal arrangement and defined pore size, coexisting with the remaining crystalline phase. The generation of mesopores would cause a decrease in the $\mathrm{Si} / \mathrm{Al}$ ratio, accompanied by an increase of the surface area with respect to the starting material. The Si species are selectively extracted, although Al species are also removed. An increase in pore diameter and mesopore volume was also observed, with the microporous structure of the starting matrices being preserved.

Keywords: Zeolites, Mesoporosity, Characterization, Glycerol.

\section{INTRODUCCIÓN}

Las zeolitas son aluminosilicatos cristalinos microporosos, cuyo tamaño de poro está en el orden de los 0,3 a $2 \mathrm{~nm}$. Estructuralmente pueden considerarse polímeros inorgánicos cristalinos con una red tridimensional de tetraedros (T) de Si y Al, enlazados entre sí a través de átomos de oxígeno. Estas redes dan lugar a una serie de canales y cavidades de dimensiones moleculares. Además, se introduce una cierta carga negativa en la 
estructura, como consecuencia de la sustitución de iones $\mathrm{Si}^{4+}$ por iones $\mathrm{Al}^{3+}$, que es compensada por protones (ácidos de Brönsted) u otro tipo de cationes (ácidos de Lewis) que se sitúan en el interior de los poros.

Las diferentes formas en que los tetraedros se enlazan en el espacio, dan lugar a una serie de sistemas de canales en las tres dimensiones, que proporcionan las características estructurales de estos materiales.

La importancia de las zeolitas en diferentes procesos catalíticos se atribuye a sus propiedades, tales como elevada área superficial, gran capacidad de adsorción, posibilidad de incorporar especies metálicas, el tamaño de los canales y cavidades (5-12 $⿱$ A) coincidente con gran cantidad de moléculas de interés, siendo una de las propiedades más importantes su selectividad de forma. Por un lado, esto supone una gran ventaja, pero también excluye muchas reacciones, al restringir el ingreso de moléculas más voluminosas hacia los sitios activos confinados en los microporos de la red. Otro problema es la desactivación por bloqueo de los poros, causada a menudo por la formación de compuestos pesados dentro de los mismos.

Las zeolitas mesoporosas no presentan este problema, debido a que su tamaño de poro es diez veces mayor que el de los materiales microporosos, aunque su limitada acidez y baja estabilidad hidrotérmica limitan su aplicación [1].

En los últimos años ha cobrado gran importancia la introducción de mesoporosidad en matrices microporosas, con el objetivo de aumentar la efectividad en ciertas reacciones químicas, como transformaciones de biomasa en productos de interés. Los mesoporos brindan mejor acceso a los microporos de las zeolitas mejorando así la velocidad de difusión de reactivos y por tanto el rendimiento catalítico [2].

Se han reportado varios métodos para lograr este objetivo, por ejemplo: i) recristalización de las paredes de tamices mesoporosos post-síntesis [3], ii) ensamblaje de las mesoestructuras a partir de soluciones precursoras de zeolitas, usando un surfactante [4] iii) templado con carbón y cristalización generando mesoporos dentro y sobre la superficie de una matriz [5], iv) extracción de silicio en zeolitas por tratamiento con soluciones alcalinas, principalmente de $\mathrm{NaOH}[6]$, v) uso de diversos agentes meso-estructurantes, diferentes a los comúnmente usados para las estructuras mesoporosas más conocidas como SBA-15, MCM-41 y HMS [7]. Por ultimo, el método más reciente vi) utiliza un agente director de estructura zeolítica sin agente mesoestructurante [8].

En este trabajo se presentan los resultados obtenidos por tratamiento alcalino de las matrices ZSM-11, BETA e Y. La extracción de silicio mediante un post tratamiento resulta en una efectiva creación de extraporosidad en zeolitas. La temperatura y el tiempo de contacto con la solución alcalina son las variables más importantes a tener en cuenta en este tratamiento, así como también la relación Si/Al de la matriz de partida

Los mencionados materiales serán utilizados posteriormente en la valorización de glicerol hacia productos de interés industrial por medio de la reacción de oxidación en fase líquida [9].

\section{MATERIALES Y MÉTODOS}

\subsection{Preparación de los catalizadores}

Las matrices zeolíticas ZSM-11 y BETA se obtuvieron por el método hidrotérmico, usando Hidróxido de Tetrapropilamonio e Hidróxido de Tetraetilamonio como agentes directores de estructura, respectivamente. Los productos de la síntesis fueron extraídos, lavados y secados a $100^{\circ} \mathrm{C}$ durante $12 \mathrm{~h}$. Para extraer el agente plantilla, las muestras fueron desorbidas bajo atmósfera de $\mathrm{N}_{2}$ desde temperatura ambiente hasta $500^{\circ} \mathrm{C}$ y luego fueron calcinadas en aire a la misma temperatura durante $12 \mathrm{~h}$, para obtener la expresión Na-Zeolitas. La forma amonio de los catalizadores $\left(\mathrm{NH}_{4}\right.$-Zeolita) se preparó por intercambio iónico con cloruro de amonio $1 \mathrm{M} \mathrm{a} 80^{\circ} \mathrm{C}$ a partir de las zeolitas en su forma sódica. Las expresiones $\mathrm{H}$-Zeolita fueron obtenidas a partir de las formas $\mathrm{NH}_{4}$-Zeolitas por tratamiento a $500^{\circ} \mathrm{C}$, con flujo de $\mathrm{N}_{2}$ durante $8 \mathrm{~h}$ y luego calcinada a la misma temperatura por $10 \mathrm{~h}$.

\subsection{Jerarquización de las zeolitas}

La generación de mesoporosidad en los materiales zeolíticos se realizó por medio de desilicación, este método consiste en la hidrólisis del enlace $\mathrm{Si}-\mathrm{O}-\mathrm{Si}$ mediante el tratamiento en medio básico ( $\mathrm{NaOH}$, normalmente). Este procedimiento permite la formación de mesoporosidad mientras se preservan las propiedades ácidas de la zeolita. Para tal fin se trataron las distintas matrices zeolíticas Na-ZSM-11, Na-Y y Na-BETA con soluciones de $\mathrm{NaOH}(0,10,2$ y $0,3 \mathrm{M})$. La técnica consistió en poner en contacto $30 \mathrm{ml}$ de solución de $\mathrm{NaOH}$ por gramo de zeolita, mediante agitación constante y sobre un baño de agua para homogeneizar la temperatura, 
que se mantuvo a $65^{\circ} \mathrm{C}$ durante 30 minutos. El proceso se detuvo colocando el beacker en un baño de agua fría. El producto resultante se recuperó por filtración mediante vacío. Luego se dejó secar en estufa a $100^{\circ} \mathrm{C}$ durante $24 \mathrm{~h}$. Posteriormente, cada una de estas muestras fueron intercambiadas 3 veces, con una solución de $\mathrm{NH}_{4} \mathrm{Cl}(0,5 \mathrm{M})$ durante $1 \mathrm{~h}$ a una temperatura de $80^{\circ} \mathrm{C}$. Después, se dejó que el sólido decante y se retiró el $\mathrm{NH}_{4} \mathrm{Cl}$ sobrenadante, reponiendo la misma cantidad para continuar con los sucesivos intercambios. El catalizador fue recuperado por filtración y secado en estufa por $24 \mathrm{~h}$. Finalmente, las muestras se trataron térmicamente en atmósfera de $\mathrm{N}_{2}(10 \mathrm{ml} / \mathrm{min})$ desde temperatura ambiente a $500^{\circ} \mathrm{C}$ y se calcinaron a la misma temperatura durante $8 \mathrm{~h}$, obteniendo de esta manera las formas H-ZSM-11(T), H-Y (T). En el caso de H-BETA (T), se logró mediante un tratamiento menos agresivo al mencionado anteriormente, que se llevo a cabo a menor temperatura $\left(45^{\circ} \mathrm{C}\right)$ y menor tiempo de contacto con el $\mathrm{NaOH}(10 \mathrm{~min})$. Este tratamiento más leve fue seleccionado debido a que el material no pudo ser recuperado por filtración después del tratamiento, indicando pérdida de la estructura cristalina.

\subsection{Caracterización de catalizadores}

Los materiales catalíticos fueron caracterizados a través de diversas técnicas:

- Difracción de rayos X (DRX) en un difractómetro Philips PW 3020 empleando radiación Ka de Cu, de longitud de onda $0,15418 \mathrm{~nm}$. Los datos de difracción se recogieron entre $2 \theta=2-60^{\circ}$, a intervalos de $0,1^{\circ}$ y velocidad de $2^{\circ}$ por min. La utilización de DRX también fue necesaria para la confirmación de estructura, grado de cristalinidad y pureza de las matrices.

- Determinación de Área Superficial por método BET fue llevada a cabo usando un equipo Micromeritics ASAP 2000 con absorción de $\mathrm{N}_{2}$ a $77 \mathrm{~K}$. Se estudió el área expuesta de las matrices de partida, luego de los tratamientos químicos y térmicos, y finalmente luego de las diversas aplicaciones.

- Las Isotermas de Adsorción de $\mathrm{N}_{2}$ a $77 \mathrm{~K}$ fueron realizadas en un equipo Micromeritics apparatus Model ASAP 2010. Se determinó la distribución de diámetro y volumen de mesoporos $\left(\mathrm{V}_{\text {meso }}\right)$ siguiendo el método BJH. El volumen total del poro $(\mathrm{Vp})$ fue determinado a una presión relativa del gas $\left(\mathrm{p} / \mathrm{p}_{0}\right)=0,98$, asumiendo un llenado completo de los poros. El volumen de los microporos $\left(\mathrm{V}_{\text {micro }}\right)$, se calculó como la diferencia entre el volumen total del poro y el volumen de mesoporos $\left(\mathrm{V}_{\text {micro }}=\mathrm{V}_{\mathrm{p}}-\mathrm{V}_{\text {meso }}\right)$.

- Espectroscopía de Emisión Atómica con Plasma Inductivamente Acoplado (ICP) en un equipo Varian 715ES. Se determina la relación molar $\mathrm{Si} / \mathrm{Al}$ que conforman la estructura cristalina de las muestras.

- Microscopía de Barrido Electrónico (SEM) se realizaron usando un microscopio Zeiss Sigma para determinación de morfología, tamaño de partículas y composición química superficial.

\section{RESULTADOS Y DISCUSIÓN}

\subsection{Difracción de Rayos $\mathrm{X}$}

En la Fig.1 se muestran los patrones de difracción de Rayos X de las zeolitas y sus correspondientes muestras sometidas a tratamiento alcalino, observándose la elevada cristalinidad y pureza de las zeolitas de partida. En los difractogramas de las muestras tratadas se puede observar la presencia de señales a bajos ángulos atribuibles a la generación de estructura hexagonal. Por otra parte, se pudo detectar una pequeña disminución de la cristalinidad a mayor concentración de $\mathrm{NaOH}$ en la solución utilizada en el proceso de desilicación, así mismo se puede ver que se mantiene la estructura cristalina de las muestras luego del tratamiento con $\mathrm{NaOH}$ 0,3M. Se detectó un pequeño ensanchamiento de la base de los picos que indicarían la presencia de material amorfo. Este comportamiento puede ser relacionado con la disolución de una parte de la zeolita requerido para la formación de la fase mesoporosa, tal como indica Qi et al. [10]. Para el caso particular de la zeolita Y, se debió realizar una modificación al tratamiento térmico posterior de las muestras sometidas a tratamiento alcalino, ya que se detectó una disminución en la intensidad de los picos característicos, a medida que el tratamiento alcalino fue más agresivo, con destrucción de la red zeolítica. Por lo que se infirió que el tratamiento alcalino de la zeolita $\mathrm{Y}$ conduce a la destrucción rápida de los enlaces $\mathrm{Si}-\mathrm{O}-\mathrm{Si}$ acompañado de una pronta desilicación y el intercambio parcial de los protones por cationes $\mathrm{Na}^{+}$, seguido de la creación de mesoporos de 3 a $20 \mathrm{~nm}$ y > $30 \mathrm{~nm}$. Se decidió entonces para poder controlar el impacto de la extracción de Si de la red, realizar los tratamientos térmicos en condiciones menos agresivas a $500^{\circ} \mathrm{C}$ durante $5 \mathrm{~h}$, de esta manera si bien la intensidad de los picos característicos se vieron disminuidos se mantuvo la estructura zeolítica. 


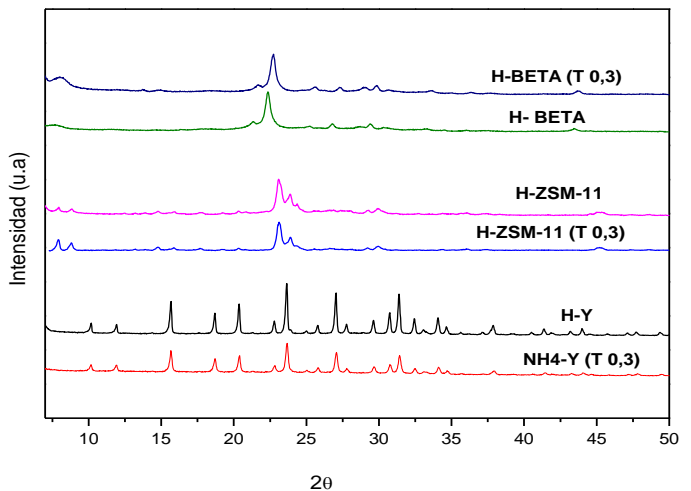

Figura 1: Patrones de DRX de las zeolitas con tratamiento alcalino 5-50 $(2 \theta)$.

\subsection{Estudio de las Isotermas de Adsorción}

Las isotermas de las zeolitas sintetizadas (ZSM-11, BETA) e Y (comercial), mostradas en la Fig. 2 corresponden a isotermas Tipo I (según la clasificación IUPAC) características de estructuras microporosas; mientras que las zeolitas tratadas, exhiben isotermas combinadas Tipo I-IV, con una mayor adsorción a p/ $\mathrm{p}_{0}$ entre 0,4-1,0, lo que indicaría la existencia de huecos entre partículas con forma irregular con amplia distribución de tamaño. Las ramas paralelas de adsorción-desorción de $\mathrm{N}_{2}$ también indicarían la presencia de canales más abiertos similares a mesoporos en las muestras tratadas [11].

El efecto de la desilicación en la estructura de poro de las zeolitas tratadas se pudo ver claramente en las isotermas de adsorción de las muestras sometidas al tratamiento alcalino. La pendiente de las isotermas de las zeolitas tratadas es mayor que las correspondientes a las de partida. 

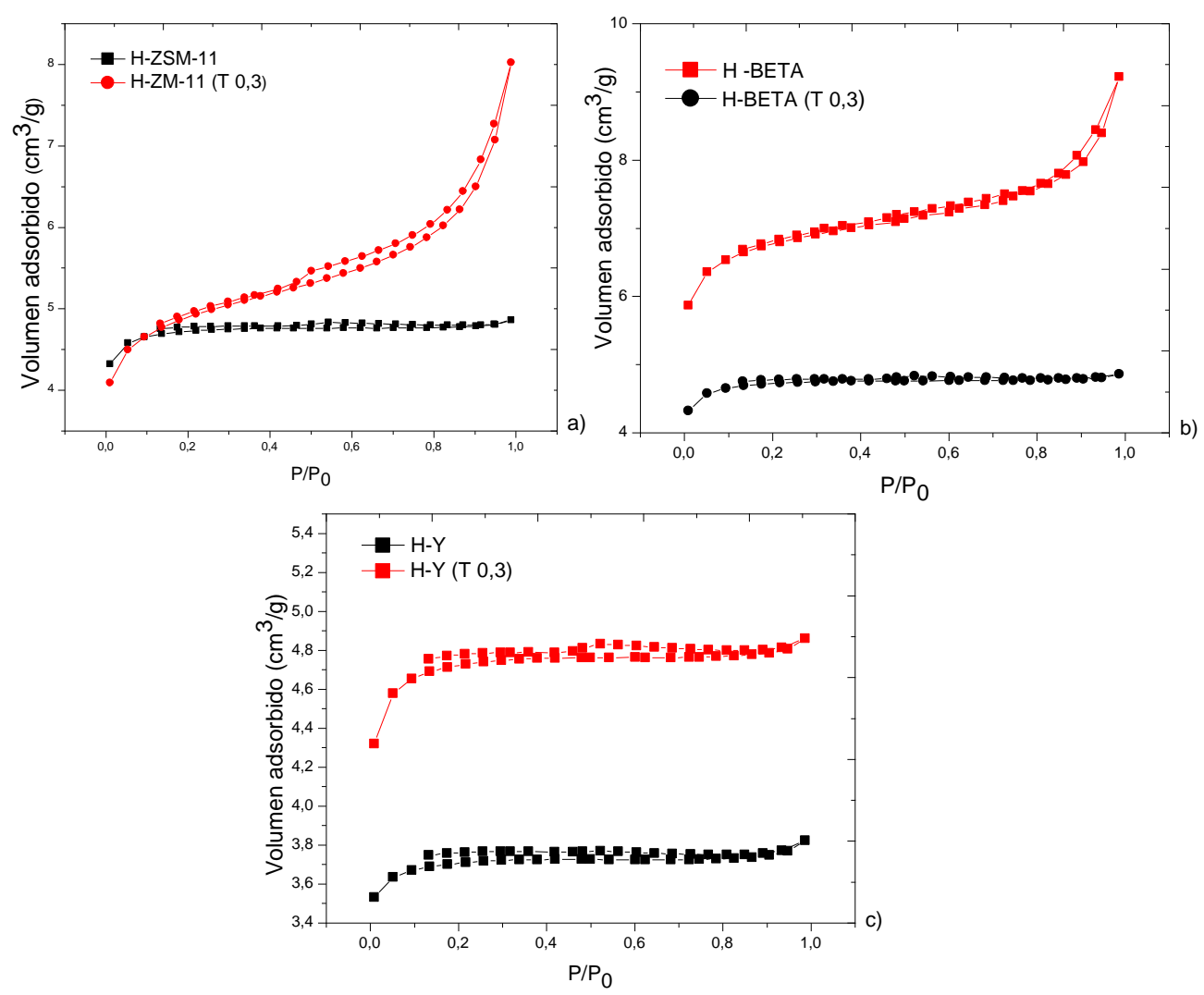

Figura 2: Isotermas de adsorción-desorción de $\mathrm{N}_{2}$ a $77 \mathrm{~K}$ de las zeolitas.

Mediante la aplicación del método BJH se obtuvieron las distribuciones de tamaños de poro de cada una de las zeolitas tratadas y sus respectivas matrices (Fig 3). La zeolita H-ZSM-11 presenta una distribución de tamaño de poro menor a $2 \mathrm{~nm}$, mientras que la zeolita tratada muestra una amplia distribución, entre 5 y $20 \mathrm{~nm}$, centrada en $10 \mathrm{~nm}$. Algo similar se observa para la matriz H-BETA, que presenta una distribución característica de un material microporoso (menor a $2 \mathrm{~nm}$ ) mientras que la zeolita tratada con $\mathrm{NaOH}$ presenta una estrecha distribución entre 2 y $5 \mathrm{~nm}$, centrada en $3 \mathrm{~nm}$. Para el caso de la zeolita $\mathrm{Y}$, no se observan variaciones en la distribución de tamaños de poros entre la muestra tratada y el material de partida. 

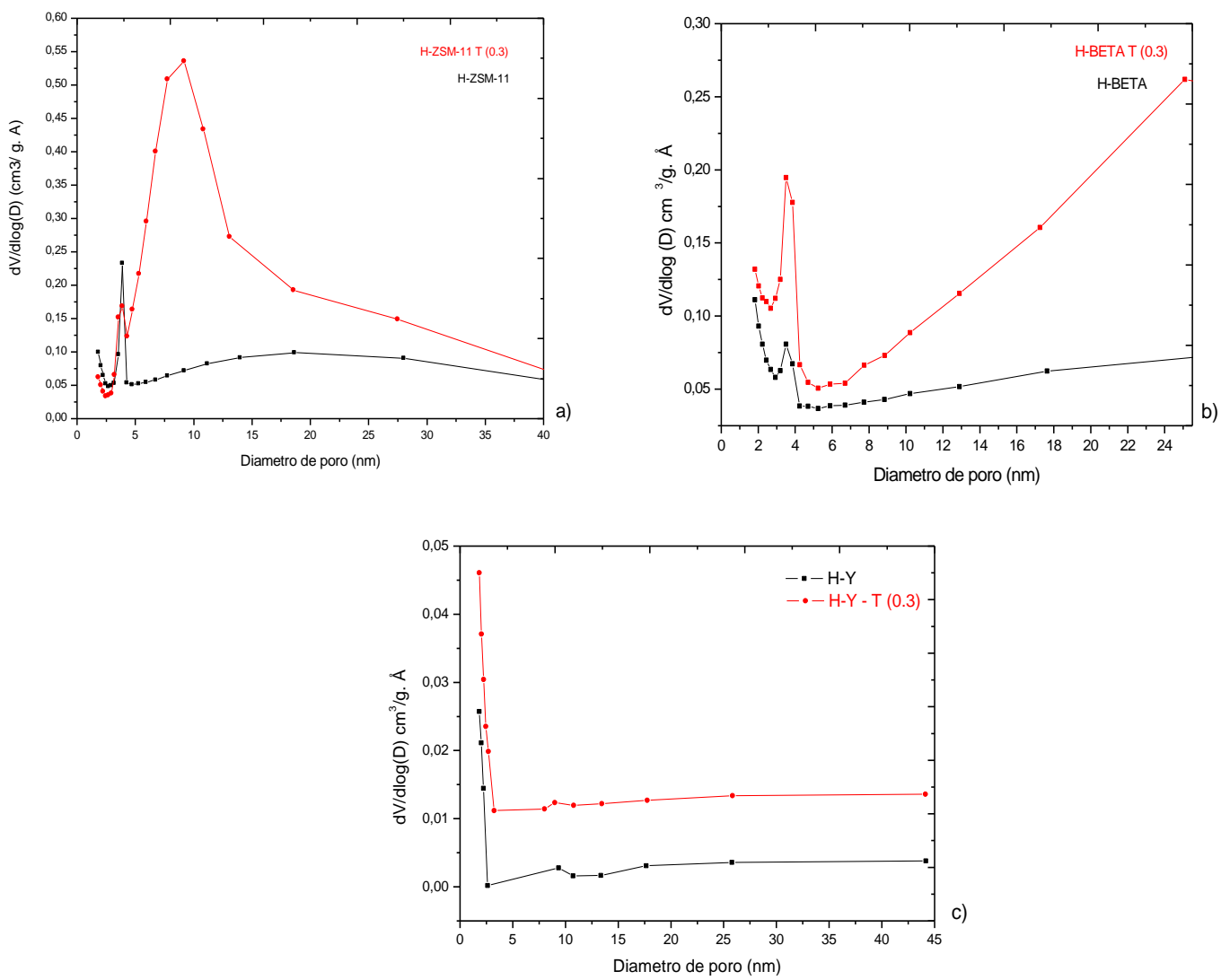

Figura 3: Distribución de tamaño de poro por $\mathrm{BJH}$ a partir de la rama de desorción de $\mathrm{N}_{2}$ de las muestras con tratamiento alcalino: a) H-ZSM-11 T $(0,3)$ b) H-BETA T $(0,3)$ c) H-Y -T $(0,3)$.

La relación $\mathrm{Si} / \mathrm{Al}$ y las propiedades estructurales de las zeolitas modificadas se pueden observar también en la Tabla 1.

Tabla 1: Propiedades texturales de las zeolitas.

\begin{tabular}{|l|l|l|l|l|l|l|l|}
\hline Zeolita & $\mathrm{Si} / \mathrm{Al}{ }^{(1)}$ & $\begin{array}{l}\mathrm{S}_{\text {BET }} \\
\left(\mathrm{m}^{2} / \mathrm{g}\right)\end{array}$ & $\begin{array}{l}\mathrm{S}_{\text {ext }} \\
\left(\mathrm{m}^{2} / \mathrm{g}\right)\end{array}$ & $\begin{array}{l}\mathrm{V}_{\text {micro }} \\
\left(\mathrm{cm}^{3} / \mathrm{g}\right)\end{array}$ & $\begin{array}{l}\mathrm{V}_{\text {meso }} \\
\left(\mathrm{cm}^{3} / \mathrm{g}\right)\end{array}$ & $\begin{array}{l}\mathrm{Vp}^{3} \\
\left(\mathrm{~cm}^{3} / \mathrm{g}\right)\end{array}$ & Dp (nm) \\
\hline Y & 2,41 & 485 & 6,1 & 0,124 & 0,006 & 0,130 & 1,99 \\
Y-T(0.3) & - & 587 & 11,9 & 0,156 & 0,010 & 0,166 & 2,01 \\
\hline ZSM-11 & 18,04 & 392 & 74,0 & 0,106 & 0,139 & 0,245 & 2,75 \\
ZSM-11-T(0.3) & - & 432 & 147,0 & 0,061 & 0,314 & 0,376 & 4,22 \\
\hline BETA & 17,40 & 585 & 67,0 & 0,180 & 0,110 & 0,291 & 2,39 \\
BETA-T(0.3) & - & 601 & 131,0 & 0,040 & 0,318 & 0,359 & 2,92 \\
\hline
\end{tabular}

(1) Análisis por ICP en estudio al momento de la presentación de este trabajo.

El área superficial de las zeolitas con tratamiento, determinado por el método BET, y la superficie externa se incrementaron en todos los casos luego del tratamiento alcalino. La relación Si/Al de las zeolitas tratadas depende de la relación de la zeolita de partida, por lo que es una variable muy importante a considerar. En primera instancia se comenzó el estudio con zeolitas BETA, ZSM-11 e Y con relación Si/Al 18, 17 y 
2,41; respectivamente. El Al en la red determina la susceptibilidad de la zeolita hacia la extracción de Si y actúa como un agente director de mesoporos en el mecanismo de la formación de poros durante la desilicación en medio alcalino [2,8].

El tratamiento alcalino de la zeolitas ZSM-11, BETA e Y, de estructura tipo MEL, BEA y FAU respectivamente, extrae de forma selectiva el Silicio. Esto conduciría a una disminución en la relación $\mathrm{Si} / \mathrm{Al}$ de las mismas.

En cuanto a las propiedades texturales, puede verse claramente un incremento en la mesoporosidad (volumen y superficie de mesoporos) en las tres matrices zeolíticas, al mismo tiempo se produce una pérdida en la microporosidad de las zeolitas, la cual es más marcada para el caso de las zeolitas ZSM-11 y BETA.

Los tratamientos realizados para generar mesoporosidad producen un aumento notable en el área específica $\left(\mathrm{S}_{\mathrm{BET}}\right)$ y en el volumen poroso de los sólidos, principalmente por un aumento notable en el volumen de mesoporos. De acuerdo a los patrones de DRX y a la distribución de poros se logra obtener sólidos en el límite inferior del intervalo definido para la clasificación de mesoporosos. El tratamiento alcalino realizado a la zeolita Y-T $(0,3)$ no generó mesoporosidad apreciable, logrando una mayor área específica y volumen de poro, de hecho se sigue obteniendo una isoterma tipo I muy similar a la obtenida para la zeolita Y de partida. En este caso, observamos que el tratamiento térmico aplicado posteriormente al tratamiento alcalino (desorción - calcinación) resultó muy agresivo destruyendo la estructura cristalina, lo cual fue observado por DRX; por lo tanto se optó por una calcinación a $500^{\circ} \mathrm{C}$ por $5 \mathrm{~h}$.

Las zeolitas de partida (ZSM-11, BETA e Y) y las muestras desilicadas han sido analizadas por SEM. En la Fig. 4 se pueden observar las zeolitas de partida (a la izquierda) y las muestras tratadas (a la derecha). La morfología de las zeolitas aparece bien definida para las matrices sin tratamiento alcalino; mientras que para el caso de las zeolitas ZSM-11 y BETA se puede observar, luego del tratamiento con la solución 0,3M de $\mathrm{NaOH}$, la presencia de cristales y material amorfo (más marcado para el caso de la zeolita BETA), lo cual se condice con los resultados de DRX. Para el caso de la zeolita Y no se evidencian cambios morfológicos en la estructura del material, lo que hace suponer que el tratamiento de desilicación no ha sido favorable, siendo adjudicable dicho comportamiento a la baja relación Si/Al $(2,41)$ de la matriz de partida. Groen et al. [12] reportan el papel del Al en el proceso de extracción de Si en zeolitas del tipo ZSM-5, donde la presencia de altas concentraciones de Al previenen la extracción de Si limitando de esta manera la formación de poros de mayor tamaño.

Las imágenes SEM muestran que el tratamiento alcalino conlleva a una reducción del tamaño de las partículas de la zeolita, aparecen defectos y grietas sobre el cristal, permaneciendo invariable la morfología de las mismas.
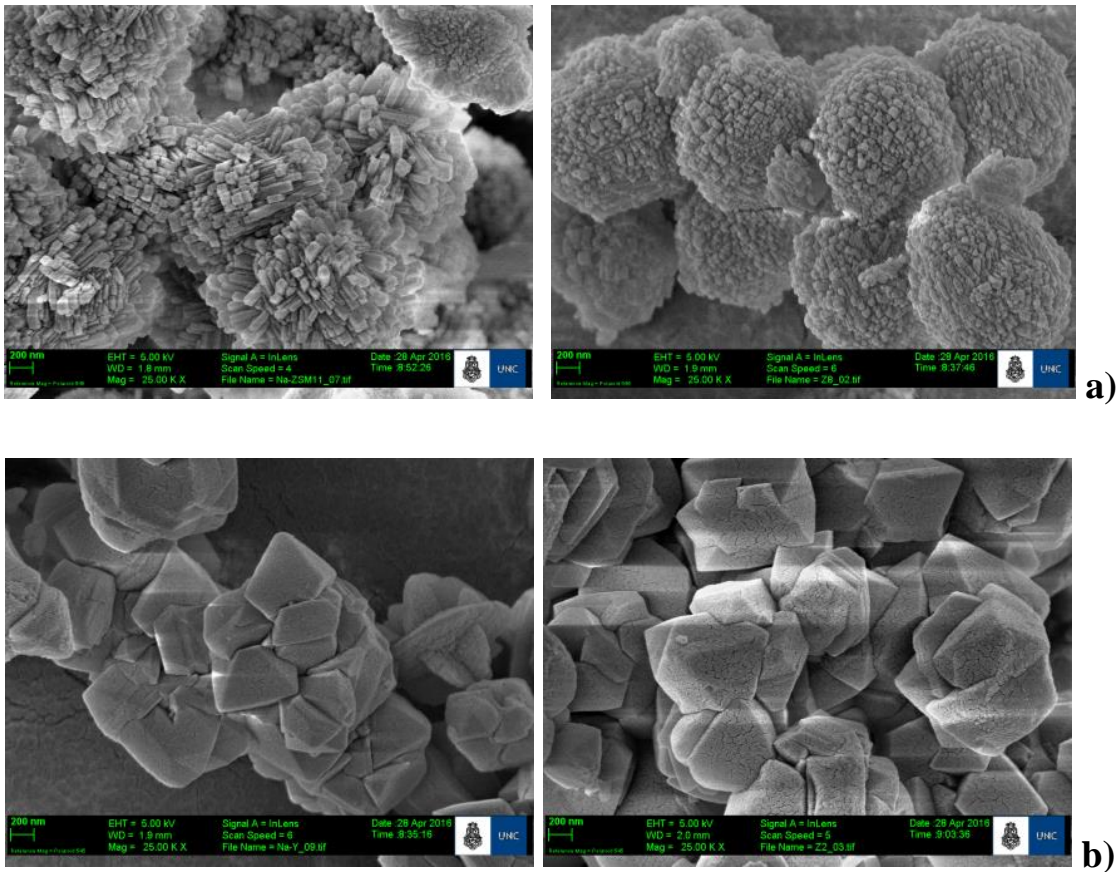
Figura 4:
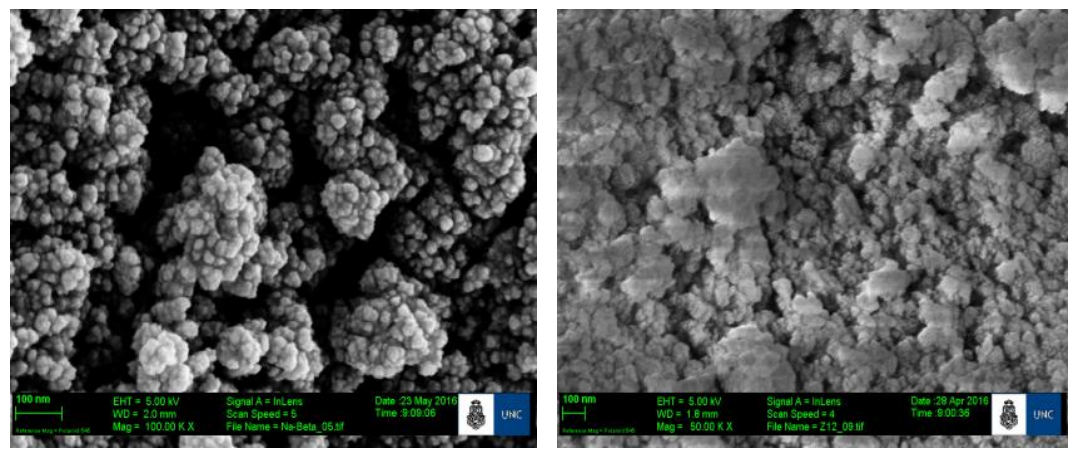

C)

de las zeolitas. a) ZSM-11, b) Y, c) BETA. Izquierda: material de partida. Derecha: material luego del tratamiento alcalino.

\section{CONCLUSIONES}

En este trabajo se ha estudiado la modificación en las propiedades y morfología de las zeolitas Y, ZSM-11 y BETA, luego del tratamiento alcalino de desilicación. Los materiales fueron caracterizados por DRX, demostrando que se mantiene la estructura cristalina después del tratamiento, observándose también, los picos característicos de la estructura hexagonal del tipo MCM-41 a bajos ángulos. También se detectó un pequeño ensanchamiento de la base de los picos que indicaría la presencia de material amorfo, requerido para la formación de la fase mesoporosa.

Mediante el estudio de Isotermas de Adsorción de $\mathrm{N}_{2}$ de las zeolitas sintetizadas (ZSM-11, BETA) y comercial (Y), se pudo observar que corresponden a isotermas Tipo I, características de estructuras microporosas, mientras que las zeolitas tratadas exhibieron isotermas combinadas Tipo I-IV, con mayor adsorción a $\mathrm{p} / \mathrm{p} 0=0,4$ a 1,0 .

El área superficial determinada por método BET, en todos los casos aumentó con respecto a la zeolita de partida. Por el método BJH obtuvimos la distribución de tamaños de poros, mostrando una amplia distribución de tamaños entre 2 y $20 \mathrm{~nm}$, lo que concuerda con el aumento en el volumen de mesoporo. De acuerdo a los resultados previamente discutidos, el tratamiento fue más exitoso para la zeolita ZSM-11 en primer lugar seguido por la BETA. En el caso particular de la zeolita Y, este tratamiento no generó un aumento en el diámetro de poro apreciable, así como del volumen de mesoporo, ésto podría deberse a la baja relación $\mathrm{Si} / \mathrm{Al}$ $(2,4)$ de la matriz de partida, ya que este es un factor muy importante para la generación de mesoporosidad.

De las imágenes SEM de la zeolita ZSM-11 fue posible apreciar la aparición de grietas y fallas en la superficie del cristal y para el caso particular de la zeolita BETA se pudo visualizar algo de material amorfo, mientras que la morfología de las mismas se mantiene. La zeolita Y no evidenció cambios en su morfología.

Se han enviado a analizar por ICP las muestras con tratamiento alcalino, no obteniéndose los resultados al momento de la presentación de este trabajo. Se pretende continuar con el estudio de las propiedades de las zeolitas micro/mesoporosas mediante otras técnicas de caracterización.

\section{AGRADECIMIENTOS}

A CONICET PIP Cod.: 11220130100146 CO (2015-2018), a UTN PID-UTN3493 A CONICET: L. Pierella, M. Renzini, C. Leal Marchena, A. Córdoba y E. Diguilio, y a UTN: L.Pierella, M. Renzini.

\section{BIBLIOGRAFÍA}

[1] CORMA, A. "State of the art and future challenges of zeolites as catalysts", Journal of Catalysis, v. 216, pp. 298-312, 2003.

[2] GROEN, J.C., ABELLO, S., VILLAESCUSA, L. A., et al., "Mesoporous beta zeolite obtained by desilication”, Microporous and Mesoporous Materials, v. 114, n. 1, pp. 93-102. 2008.

[3] KLOETSTRA, K.R., VAN BEKKUM, H., CANSEN, J.C, "Mesoporous material containing framework tectosilicate by pore-wall recrystallization”, Chemical Communcation, v. 23, pp. 2281-2282, 1997.

[4] LIU, Y., ZHANG, W., PINNAVAIA, T.J, "Steam-Stable Aluminosilicate Mesostructures Assembled from Zeolite Type Y Seeds”, Journal of the American Chemical Society, v. 122, pp. 8791-8792, 2000. 
[5] JACOBSEN, C.J.H., MADSEN, C., HOVZVICKA, J., et al., "Mesoporous Zeolite Single Crystals", Journal of the American Chemical Society, v. 122, pp. 7116-7117, 2000.

[6] OGURA, M., SHINOMIYA, S., TATENO, J., et al., "Alkali-treatment technique - new method for modification of structural and acid- catalytic properties of ZSM-5 zeolites", Applied Catalysis A: General, v. 219, pp. 33-43, 2001.

[7] ZHANG, B., DAVIS, S., MANN, S. "Starch gel Templating of Spongelike Macroporous Silicalite Monoliths and Mesoporous Films", Chemistry ofl Materials, v. 14, pp. 1369-1375, 2002.

[8] LÓPEZ, C. M., SAZO, V., URBINA, C., et al., "Tamices molecualres que combinan micro y mesoporosidad: una revisión", Avances en Química, v. 4, n. 3, pp. 113-125, 2009.

[9] GROEN, J. C., JANSEN, J. C., MOULIJN, J. A., et al., "Optimal Aluminum- Assisted Mesoporosity Development in MFI Zeolites by Desilication", The Journal of Phisical Chemistry B, v. 108, pp. 1306213065, 2004

[10] QI, J., ZHAO, T., XU, X., et al., "Study of cracking of large molecules over a novel mesoporous Beta", Catalysis Communications, v. 10, n. 11, pp. 1523-1528, 2009.

[11] HUI, L., SHENGLIN, L., SUJUAN, X., et al., "Effect of Desilication on the Performance of Hierarchical ZSM-11 Catalysts for Alkylation of Benzene with Dimethyl Ether", Catalisys Letter, v. 145, n 11, pp. $1972-1983,2015$.

[12] GROEN, J., MOULIJN, J., PÉREZ RAMÍREZ, J, "Decoupling mesoporosity formation and acidity modification in ZSM-5 zeolites by sequential desilication - dealumination", Microporous and Mesoporous Materials, v. 87, n. 2, pp. 153-161, 2005. 